\title{
Black Holes in Galactic Nuclei, X-Ray Binaries and Ultraluminous X-Ray Sources
}

\author{
Zdenka Kuncic ${ }^{\mathrm{A}}$ \\ A School of Physics, University of Sydney, Sydney NSW 2006, Australia. \\ Email: z.kuncic@physics.usyd.edu.au
}

Received 2004 December 23, accepted 2005 March 21

\begin{abstract}
This review summarizes the astrophysical evidence for the existence of black holes provided by their gravitational influence on nearby matter. Two classes of accreting black holes have now been observationally verified: supermassive black holes (SMBHs) in galactic nuclei, and stellar-mass black holes in X-ray binaries (XRBs). With the recent re-discovery of ultra-luminous X-ray (ULX) sources, fresh evidence has also emerged for the existence of a third class of accreting black holes: intermediate-mass black holes (IMBHs). The properties of the three classes of accreting black holes are briefly discussed.
\end{abstract}

Keywords: black holes — accretion — galaxies: nuclei - X-rays: binaries

\section{Introduction}

The existence of cosmic entities, whose gravitational field is so strong that not even light can escape its powerful grasp, was first speculated upon over two centuries ago by mathematicians John Michell and Pierre-Simon Laplace. These speculations were theoretically vindicated in 1916, when Karl Schwarzschild solved Einstein's field equations for the limiting case of a point mass in vacuo. In 1930, Subrahmanyan Chandrasekhar calculated that, once all their nuclear fuel is exhausted, stars more massive than $1.4 \mathrm{M}_{\odot}$ will never attain a stable gravitational equilibrium state in the form of a white dwarf. This laid the theoretical groundwork for modern stellar evolution theory, according to which stars more massive than $\sim 3 \mathrm{M}_{\odot}$ are predestined to live out the end of their lives as a black hole.

The first tentative observational evidence for the existence of black holes emerged with the advent of the first large radio and optical telescopes in the mid-twentieth century. It immediately became apparent that some cosmic radio sources exhibit energetic phenomena that are incompatible with activity of a stellar origin. In the late 1950s, Geoffrey Burbidge showed that the total energy output from the extended lobes of radio galaxies such as Cygnus A vastly exceeds even that of a supernova explosion (Burbidge 1956, 1959). The next empirical breakthrough came with the cosmological interpretation of the systematic redshifts associated with optical spectral features in quasi-stellar objects (QSOs), which confirmed that these sources, like radio galaxies, are also intrinsically powerful and require an extraordinary fuelling mechanism (Greenstein \& Schmidt 1964).

At around the same time, theoretical efforts were being made towards determining observational signatures of a black hole's gravitational influence on nearby matter. Zel'dovich \& Novikov (1964) proposed that stellar-mass black holes could be potentially detected if they reside in a close interacting binary system, where matter from the companion star could radiate its gravitational potential energy as it accretes onto the black hole (see also Salpeter 1964). These theoretical ideas were then extended to the context of galactic nuclei harbouring a supermassive $\left(10^{6}-10^{9} \mathrm{M}_{\odot}\right)$ black hole remnant accreting vast quantities of matter from the intergalactic medium (Lynden-Bell 1969; see also Lynden-Bell \& Rees 1971).

Dramatic technological developments then ushered in a new era in observational astronomy in the 1970s, when cosmic sources became detectable for the first time at X-ray energies. The first X-ray satellite, Uhuru, immediately revealed a whole new class of astrophysical sources, XRBs, and provided the first convincing observational evidence for the existence of stellar-mass black holes with the discovery of Cygnus X-1. Shakura \& Sunyaev (1973) were the first to explain why stellar-mass black holes in close interacting binary systems are X-ray sources, with their formulation of a detailed theory for disk accretion onto a black hole (see also Pringle \& Rees 1972; Novikov \& Thorne 1973). Shakura \& Sunyaev (1973) showed that the structure and radiative properties of accretion disks around black holes depend primarily on the mass accretion rate, $\dot{M}_{\mathrm{a}}$. The success of the standard ' $\alpha$-disk' model of Shakura \& Sunyaev rests on the simplifying assumption that the viscous stresses $t_{r \phi}$ responsible for angular momentum transport are most likely turbulent in nature, and can be prescribed as $\alpha P$, where $P$ is the local pressure and $\alpha$ is a global constant.

In recent years, some of the most compelling evidence for the existence of accretion disks around SMBHs in galactic nuclei comes from high-resolution X-ray spectroscopy. Figure 1 shows the XMM-Newton spectrum of a broad $\mathrm{Fe} \mathrm{K} \alpha$ emission line $(6.4 \mathrm{keV}$ rest-frame 


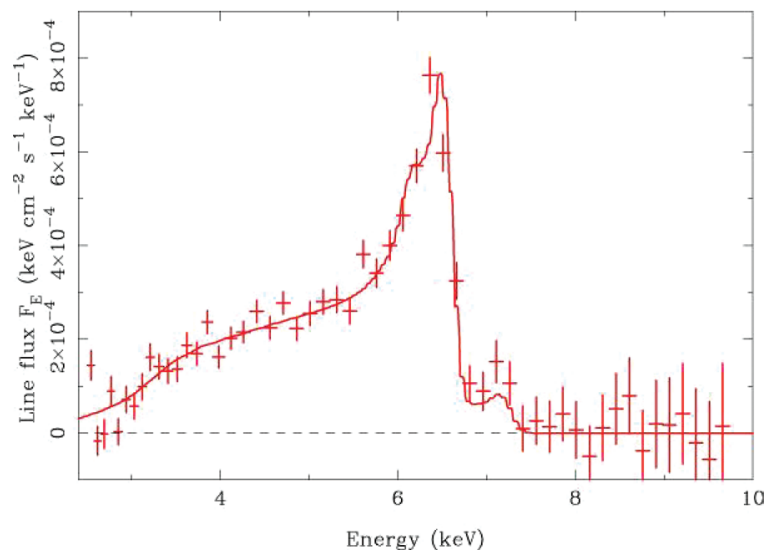

Figure 1 Spectrum of the iron K $\alpha$ line in MCG-6-30-15 taken with XMM-Newton (crosses indicate data, solid line indicates the best-fit model), showing the combined effects of gravitational redshift and relativistic Doppler shifts (from figure 3 in Fabian et al. 2002).

energy) from the active galaxy MCG-6-30-15 (Fabian et al. 2002; see also Tanaka et al. 1995). The extremely broad, asymmetric line profile is consistent with the interpretation that the emission arises from matter accreting with quasi-Keplerian angular motions in a relativistic potential. Indeed, the best-fit model for the Fe line shown in Figure 1 is one which includes the effects of gravitational redshift and relativistic transverse Doppler shifts from regions extending down to $3 r_{\mathrm{s}}$ (where $r_{\mathrm{s}}=2 G M_{\bullet} / c^{2}$ is the Schwarzschild radius of a black hole of mass $M_{\bullet}$ ).

Notwithstanding these exciting results from state-ofthe-art X-ray space telescopes, the most credible evidence for the existence of accreting SMBHs still remains the high-precision dynamical mass estimates obtained from proper motions and radial velocities of water megamaser line emission. These measurements were first obtained from VLBA observations of the nearby Seyfert II galaxy NGC 4258, and imply a central binding mass of $(3.5 \pm 0.1) \times 10^{7} \mathrm{M}_{\odot}$ within a radius of just $0.13 \mathrm{pc}$ (Miyoshi et al. 1995; see also Figure 2). Water megamaser observations also imply that accretion disks within $1 \mathrm{pc}$ of a central SMBH are extremely geometrically thin (i.e. $h / r \ll 1)$.

Unique measurements of the dynamical centre of our own Milky Way galaxy have been made by tracking the orbits of individual stars (see Figure 3). From the proper motions and radial velocities of these stars, it has been inferred that the position of the compact, nonstellar source $\mathrm{Sgr} \mathrm{A}^{\star}$ is coincident with a dark, localized mass of (3-4) $\times 10^{6} \mathrm{M}_{\odot}$ that can only be attributed to a black hole (Ghez et al. 2003).

One of the most significant recent developments in standard accretion disk theory has been the demonstration, via numerical simulations, that magnetohydrodynamic (MHD) turbulence can transport angular momentum out to large radii, thus explaining how accretion works (see Balbus 2003, for a review). However, one critical observational property of accreting black holes that is yet to be fully explained is the ubiquity of outflow phenomena,

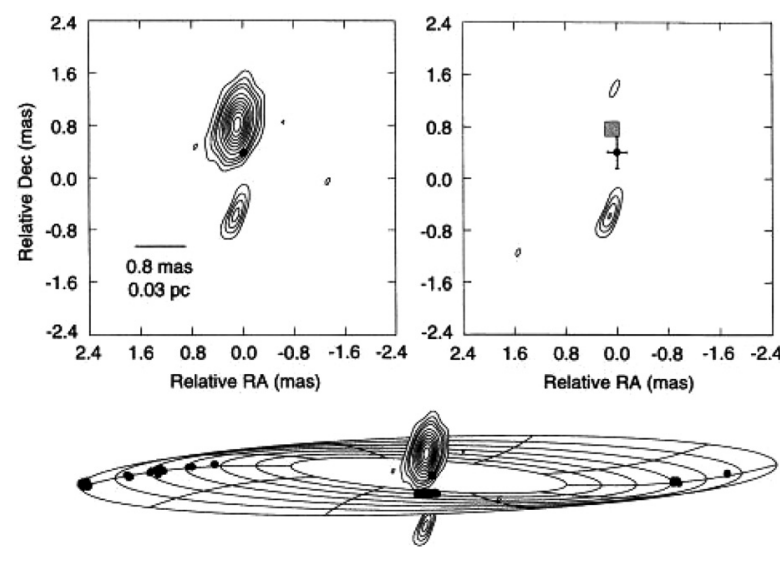

Figure 2 VLBA $22 \mathrm{GHz}$ continuum image of NGC 4258 (upper left panel), the continuum image after deconvolving the northern jet emission (upper right panel), and the continuum map superimposed on a best-fit warped accretion disk model with the locations of masing source sites indicated by filled circles and the dynamical centre indicated by a filled square (lower panel) (from figure 1 in Herrnstein et al. 1998).

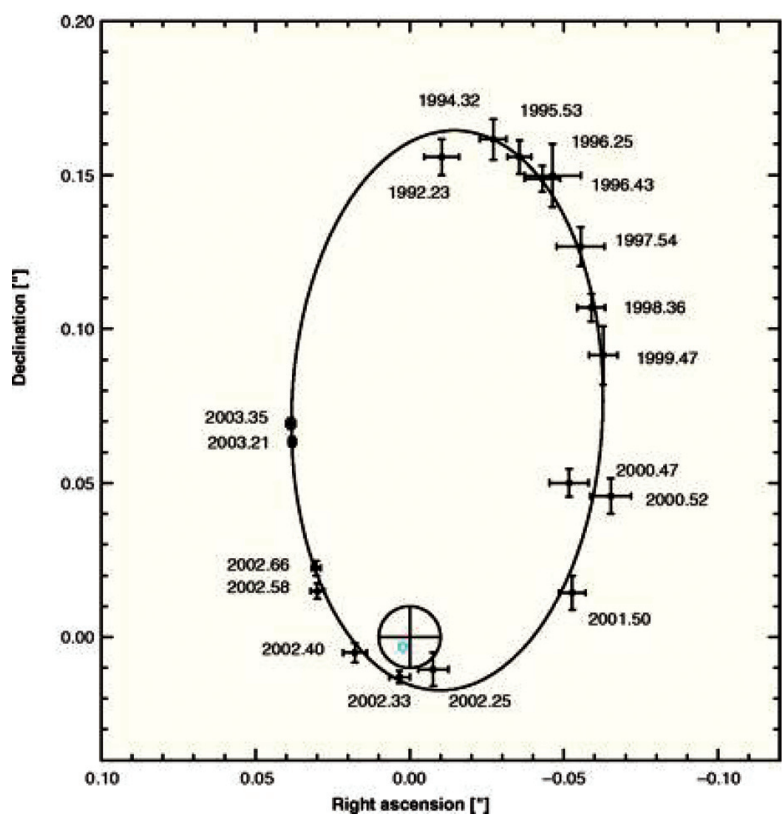

Figure 3 Orbit of the star S2 around $\mathrm{Sgr} \mathrm{A}^{\star}$ (marked by the large circled cross). The crosses denote position measurements (with $1 \sigma$ error bars) obtained from near infrared imaging observations. The solid continuous curve shows the best-fit Keplerian orbit, corresponding to a central dynamical mass of $3.6 \pm 0.6 \times 10^{6} \mathrm{M}_{\odot}$. The focus of the ellipse is indicated by the small open circle and is within a few milliarcseconds (or $2000 r_{\mathrm{s}}$ ) from Sgr A* (from figure 2 in Eisenhauer et al. 2003).

which is indicative of non-radial (i.e. vertical) momentum and energy transport (see Kuncic \& Bicknell 2004, for recent theoretical results).

Finally, recent X-ray observations have re-discovered what is now recognized as a new class of accreting black hole sources: ultraluminous X-ray sources (ULXs). These sources are particularly interesting because there is strong evidence, at least in some cases (see Soria et al. 2004), for 
the power source being attributable to an intermediatemass $\left(100-1000 \mathrm{M}_{\odot}\right.$ ) black hole (see Miller \& Colbert 2004 , for a review).

The remainder of this review paper is organized as follows: Section 2 discusses supermassive black holes in galactic nuclei; Section 3 discusses stellar-mass black holes in XRB; Section 4 discusses intermediate-mass black holes in ultraluminous X-ray sources; and a summary is presented in Section 5.

\section{Galactic Nuclei}

SMBHs residing at the centres of galaxies are usually associated with Active Galactic Nuclei (AGN). These objects are renown for exhibiting a diverse range of energetic and dynamic phenomena which appear to originate from a powerful central source confined to only a few parsecs in size. However, a significant fraction of all galaxies (of which about $10 \%$ are classed as AGN) possess nuclei that are active at some level above that which can be identified with the thermonuclear sources of the constituent stars. Indeed, there is mounting observational evidence in support of the idea proposed over 30 years ago (LyndenBell \& Rees 1971) that most galaxies have evolved through an active 'quasar' phase in their history, and therefore must harbour a relic supermasive black hole. Such dead quasar remnants are today identified as Low Luminosity AGN (LLAGN), the best example of which is our own Galactic centre.

\subsection{Active Galactic Nuclei}

AGN are characterized by one or more of the following properties: broadband spectral energy distributions extending across the entire electromagnetic spectrum; strong spectral lines (often due to extremely highly ionized atomic species), with equivalent widths and Doppler motions considerably larger than those observed in galactic spectra; high bolometric luminosities $\left(L \sim 10^{38} \mathrm{~W}\right)$, with the most luminous being so bright that they completely swamp the light from all the stars of the host galaxy; spectacular jets ejected from the nucleus at relativistic speeds and collimated in opposite directions along the galactic rotation axis, often forming extended bipolar structures; rapid and strong spectral variability, with the fastest $\mathrm{X}$-ray light-crossing times $\left(t_{\mathrm{X}}\right.$ about a few hundred seconds) implying extremely compact, highenergy source regions.

One of the most outstanding problems in the field of AGN is how to unify the observational classification of so many sub-classes of AGN (quasars, Seyferts, radio galaxies, blazars, etc.) into a single scheme. The relative influence of intrinsic properties $\left(M_{\bullet}, \dot{M}_{\mathrm{a}}\right.$, and, in the case of Kerr black holes, the spin parameter $a$ ) and extrinsic properties (orientation, host galaxy, and evolution) still remains unclear. However, some new clues that may shed light on the AGN unification problem have emerged. Firstly, recent all-sky surveys (e.g. FIRST, 2dF) indicate that, contrary to conclusions drawn from earlier surveys (e.g. LBQS), there is no dichotomy in the radio power of quasars (see e.g. Cirasuolo et al. 2003). Secondly, there is mounting evidence from observations of individual sources for a continuum in jet power in quasars, Seyferts, and other AGN (see Blundell et al. 2003, for the discovery of a relativistic jet in a radio-quiet quasar). These observational clues suggest that the jet formation mechanism operates under a wide range of conditions and is not restricted to a special class of sources.

\subsection{Low Luminosity AGN}

It is becoming increasingly apparent that SMBHs reside not just at the centres of quasars and other bonafide AGN, but also at the centres of less luminous galaxies, such as our own Milky Way. Ho et al. (1997) found that while almost $40 \%$ of nearby bright $\left(B_{T}<12.5 \mathrm{mag}\right)$ galaxies show some evidence of AGN activity, indicative of the presence of a central $\mathrm{SMBH}$, most are highly underluminous, with $L_{\text {bol }} \lesssim 10^{-2} L_{\text {Edd }}$ (where $L_{\mathrm{Edd}}=4 \pi G M_{\bullet} m_{\mathrm{p}} c / \sigma_{\mathrm{T}}, \simeq 1.3 \times 10^{31}\left(M_{\bullet} / \mathrm{M}_{\odot}\right) \mathrm{W}$, is the Eddington luminosity). Since the luminosity is related to the mass accretion rate via $L=\varepsilon \dot{M}_{\mathrm{a}} c^{2}$, where $\varepsilon$ is the radiative conversion efficiency, this implies that either LLAGN are accreting matter at substantially below the Eddington rate, $\dot{M}_{\text {Edd }}$, or $\varepsilon$ is unusually low and hence most of the gravitational energy is being converted into forms other than radiation (e.g. bulk kinetic energy). Considerable efforts have been made towards explaining inefficient accretion flows in terms of ADAFs (Advection Dominated Accretion Flows). However, ADAF models substantially underpredict the observed radio flux from $\mathrm{Sgr}^{\star}$ and other nearby LLAGN (Anderson et al. 2004). At present, the best alternative models for LLAGN are those which invoke the presence of a compact radio jet (e.g. Falcke et al. 2004).

\section{X-Ray Binaries}

XRBs are characterized by strong and highly variable $\mathrm{X}$-ray emission. Typical $\mathrm{X}$-ray luminosities and variability timescales are $L_{\mathrm{X}} \sim 10^{31} \mathrm{~W}$ and $t_{\mathrm{X}} \sim 1 \mathrm{~ms}$, respectively. This implies an extremely compact source region, $R_{\mathrm{X}} \sim 100 \mathrm{~km}$. However, definitive evidence for the presence of a stellar-mass black hole in an interacting close binary system relies on ruling out the presence of a neutron star, and this requires establishing that the mass of the compact object is $\gtrsim 3 \mathrm{M}_{\odot}$. A lower limit on the dynamical mass can be obtained from the observed radial velocity curve of the visible companion star. Unfortunately, optical spectral lines from the companion are often quite difficult to identify, and the optical mass function can be difficult to constrain, especially in persistently bright X-ray sources. The most reliable dynamical mass estimates come from $\mathrm{X}$-ray transients observed in quiescence.

Currently, there are $\sim 17$ known black hole XRBs (Orosz 2002). Three of these (Cyg X-1, LMC X-1, LMC X-3) are high-mass X-ray binaries (HMXBs), in which the companion is typically a massive $O / B$ star. The remaining majority are transient low-mass $X$-ray binaries (LMXBs), in which the mass of the companion is $\lesssim 1 \mathrm{M}_{\odot}$. The prototype of this category is A 0600-00. There are at 
least a few dozen more sources that have been identified as tentative black hole XRBs based on their spectral and temporal X-ray properties.

\section{Ultraluminous X-Ray Sources}

ULXs are discrete sources with observed X-ray luminosities exceeding the Eddington limit for a stellar-mass black hole, i.e. $L_{\mathrm{X}}>10^{32} \mathrm{~W}$. While these sources are incompatable with galactic nuclei or supernova remnants, their true nature remains unclear. Nonetheless, two classes of sources are viable candidates: unusual black hole XRBs, and accreting intermediate-mass $\left(M_{\bullet} \sim 10^{2}-10^{3}\right)$ black holes (IMBHs). At least three different models have been proposed to explain ULXs according to the XRB paradigm: (a) HMXBs possibly with a black hole at the upper end of the stellar-mass class (i.e. $M_{\bullet} \sim 20-50 \mathrm{M}_{\odot}$ ), with mild geometrical beaming during phases of super-Eddington accretion (King \& Pounds 2003); (b) micro-quasars/blazars with strongly beamed emission due to a relativistic jet pointing towards us (Körding, Falcke, \& Markoff 2002); (c) XRBs with intrinsically super-Eddington, isotropic emission from an inhomogeneous accretion disk (Begelman 2002). A recent review of the observational properties and proposed models for ULXs is given by Soria et al. (2004).

Optical counterpart searches have revealed that the brightest ULXs tend to be found in star-forming galaxies, particularly those indicative of having undergone a recent interaction event. There is also some evidence for an association between ULXs and massive star clusters, which is consistent with proposed IMBH formation mechanisms from merger processes in a cluster core. Furthermore, while there is insufficient evidence to rule out the XRB interpretation for most ULXs, a few exceptionally bright sources $\left(L_{\mathrm{X}}>10^{33} \mathrm{~W}\right)$ are difficult to reconcile with XRBs and thus appear to require an IMBH (Colbert \& Miller 2005). The existence of an intermediatemass class of black holes has far-reaching implications for our understanding of stellar cluster dynamics, SMBH formation, and gravitational wave sources (see Miller \& Colbert 2004, for a review).

\section{Summary}

This review has summarized the astrophysical evidence for the existence of black holes, based on observational signatures of accretion. There is now a wide range of corroborating evidence, spanning different observing wavebands and different observing techniques, supporting the existence of the supermassive and stellar-mass classes of accreting black holes. There is now also convincing evidence from X-ray observations for the existence of an intermediate-mass class of accreting black holes. Arguably the single most outstanding problem that continues to challenge our understanding of accreting black holes is the jet formation mechanism.

\section{Acknowledgments}

The author thanks the organizers of the Gravity 2004 workshop for a stimulating and informative meeting.

\section{References}

Anderson, J. M., Ulvestad, J. S., \& Ho, L. C. 2004, ApJ, 603, 42

Balbus, S. A. 2003, ARA\&A, 41, 555

Begelman, M. C. 2002, ApJ, 568, L97

Blundell, K. M., Beasley, A. J., \& Bicknell, G. V. 2003, ApJL, 591, 103

Burbidge, G. R. 1956, ApJ, 124, 416

Burbidge, G. R. 1959, ApJ, 129, 849

Cirasuolo, M., et al. 2003, MNRAS, 341, 993

Colbert, E. J. M., \& Miller, M. C. 2005, in eds. M. Novello, S. PerezBergliaffa, \& R. Ruffini (Singapore: World Scientific), in press (astro-ph/0402677)

Eisenhauer, F., et al. 2003, ApJ, 597, L121

Fabian, A. C., et al. 2002, MNRAS, 335, L1

Falcke, H., Körding, E., \& Markoff, S. 2004, A\&A, 414, 895

Ghez, A. M., et al. 2003, ApJL, 586, 127

Greenstein, J. L., \& Schmidt, M. 1964, ApJ, 140, 796

Herrnstein, J. R., et al. 1998, ApJ, 497, L69

Ho, L. C., Filippenko, A. V., \& Sargent, W. L. W. 1997, ApJS, 112,315

King, A. R., \& Pounds, K. A. 2003, MNRAS, 345, 657

Körding, E., Falcke, H., \& Markoff, S. 2002, A\&A, 382, L13.

Kuncic, Z., \& Bicknell, G. V. 2004, ApJ, 616, 669

Lynden-Bell, D. 1969, Natur, 223, 690

Lynden-Bell, D., \& Rees, M. J. 1971, MNRAS, 152, 461

Miller, M. C., \& Colbert, E. J. M. 2004, IJMPD, 13, 1

Miyoshi, M., et al. 1995, Natur, 373, 127

Novikov, I. D., \& Thorne, K. S. 1973, in Black Holes, eds. C. DeWitt, \& B. DeWitt (New York: Gordon \& Breach)

Orosz, J. A. 2002, in Proc. IAU Symp. 212, eds. K. A. van der Hucht, A. Herrero, \& C. Esteban, 1

Pringle, J. E., \& Rees, M. J. 1972, A\&A, 21, 1

Salpeter, E. E. 1964, ApJ, 140, 796

Shakura, N. I., \& Sunyaev, R. A. 1973, A\&A, 24, 337

Soria, R., Cropper, M., \& Motch, C. 2004, ChJAA, in press (astro-ph/0409130)

Tanaka, Y., et al. 1995, Natur, 375, 659

Zel'dovich, Y. B., \& Novikov, I. D. 1964, Dokl. Acad. Nauk. SSSR, 158,811 